\title{
4
}

\section{Retinoblastoma - Genetic Counseling and Molecular Diagnosis}

\author{
Claude Houdayer1,4, Marion Gauthier-Villars' ${ }^{1}$, Laurent Castéra ${ }^{1}$, \\ Laurence Desjardins' ${ }^{2}$, François Doz ${ }^{3,4}$ and Dominique Stoppa-Lyonnet ${ }^{1,4,5}$ \\ ${ }^{1}$ Genetics Department, Institut Curie, Paris \\ ${ }^{2}$ Ophtalmology Department, Institut Curie, Paris \\ ${ }^{3}$ Pediatrics Department, Institut Curie, Paris \\ ${ }^{4}$ Université Paris Descartes, Paris \\ IINSERM U830, Pathologie Moléculaire des Cancers, \\ Institut Curie, Paris \\ France
}

\section{Introduction}

Retinoblastoma is a malignant embryonal tumour of childhood arising at the expense of retinal cones. It has an incidence of 1 per 15,000 to 20,000 births. In $90 \%$ of cases, it is diagnosed before the age of 3 years. The possibility of conservative management depends on early diagnosis (Moll et al., 1996). However, although treatment strategies have advanced considerably, the visual prognosis is still a major source of concern, especially central vision when the tumour is situated at or close to the macula. In two-thirds of cases, the lesion is unilateral and the median age of diagnosis is 2 years. In the other third, the lesion is bilateral and the disease is diagnosed earlier, possibly even during the neonatal period, with a median age of diagnosis of 1 year. Most cases of unilateral and bilateral retinoblastoma are sporadic, with no family history. However, 10 to $15 \%$ of all cases of retinoblastoma present a family history. The distribution of cases within the family is compatible with the existence of a tumour susceptibility gene transmitted according to an autosomal dominant mode with high penetrance. In this case, the lesion is usually bilateral and diagnosed at an early age.

\subsection{Diagnosis}

The most frequent presenting signs are leukocoria (white pupillary reflex) and strabismus. Retinoblastoma may also be discovered on routine ocular fundus examination performed in a child from a high-risk family.

The diagnosis is essentially based on the ocular fundus examination under general anaesthesia, completed by ultrasound and CT. Tumour growth may be endophytic with invasion of the vitreous cavity or, more rarely, exophytic with retinal detachment. A precise description of the lesions based on fundoscopy findings allows the lesion to be classified according to the 5 stages of the Reese-Ellsworth classification, associated with an increasingly severe prognosis. Ultrasound and orbital CT demonstrate tumour calcifications 
highly suggestive of retinoblastoma and CT contributes to staging in advanced forms. In the case of enucleation, the diagnosis and staging are confirmed by histological criteria.

Conservative treatment must be attempted whenever possible: chemotherapy, radiotherapy, photocoagulation, and cryotherapy. Very advanced forms unsuitable for conservative treatment will require enucleation. This treatment is still unfortunately often required for sporadic retinoblastomas whose diagnosis is made late.

\subsection{Predisposition to retinoblastoma}

In 1971, Knudson proposed a model designed to explain why most familial retinoblastomas were bilateral and occurred at an early age and, inversely, why unilateral cases were usually isolated and diagnosed later (Knudson, 1971). He proposed the hypothesis that two mutations of key genes in the control of cell division occurring in a retinal neuroectodermal cell were necessary, but possibly not sufficient, for development of retinoblastoma. In bilateral forms, the first mutation is a germline mutation, present in all cells of the body and especially in all retinal neuroectodermal embryonal cells, while the second mutation is somatic, acquired during foetal life or the first months of neonatal life. Although the probability of two somatic mutations in two key genes in the same retinal cell is extremely low, development of a single mutation is not a rare event and induces development of a retinoblastoma when another mutation is already present. This explains why children with a germline mutation have a high risk of developing not just one, but two or more tumours. Comings completed Knudson's hypothesis in 1973 by postulating that the two mutations necessary for the development of retinoblastoma corresponded to inactivation of the two alleles of the same gene, that had not yet been identified at that time (Comings, 1973). The hypothesis of the existence of tumour suppressor genes, already suspected, became very likely.

In familial cases, the germline mutation is transmitted by one of the parents. In sporadic, bilateral and sometimes multifocal unilateral cases, the germline mutation usually corresponds to a de novo mutation arising in the gametes of one of the two parents (prezygotic) or at an early stage after fertilization (post-zygotic). Pre-zygotic de novo mutations are associated with advanced paternal age. In some cases, the apparently sporadic nature of retinoblastoma is related to incomplete penetrance in one carrier parent. As the risk of tumour is high, but incomplete, a parent with a germline mutation may fail to develop retinoblastoma during childhood or may have developed a spontaneously regressive retinoblastoma, which may leave a retinal scar or retinoma. It is therefore very important to perform an ocular fundus examination in each parent looking for retinoma, which would reveal a previously unknown family history that would consequently modify genetic counseling. This point is discussed in more detail in the "Notes" section of the chapter on "Genetic counseling".

Most unilateral cases are due to two mutations occurring only at the somatic level. However, it is estimated that almost $10 \%$ of patients with unilateral retinoblastoma have a germline mutation.

A risk of cancer different of retinoblastoma exists within retinoblastoma predisposition. Rare patients develop pineal region tumour but is considered like an ectopic intracranial retinoblastoma and so-called trilateral retinoblastoma. An increased risk of second cancers 
for RB1 mutation carriers, after retinoblastoma, is well documented. In childhood and early adulthood, these patients have a high incidence of osteosarcomas and soft tissue sarcomas. The incidence of these cancers can often be attributed to external beam radiation therapy, but many cases have been reported occurring outside of the field of radiation treatment and even for patients who received no radiation. A cumulative rate of second cancers is reported 18 years after the diagnosis of genetic retinoblastoma at $8.4 \%$ (and 6\% for osteosarcomas alone) (Draper et al., 1986). Otherwise, RB1 mutation carriers have also a high lifetime risk of developing a late onset epithelial cancer (lung, bladder, breast) and melanoma. In a historic series of 144 survivors of hereditary retinoblastomas, the cumulative cancer incidence to 85 years of age has been estimated to be $68.8 \%$ ( $\mathrm{CI}=48.0 \%$ to $87.4 \%$ ) (Fletcher et al., 2004).

\subsection{The RB1 gene}

The identification, in 1963, of germline deletions of chromosome 13 (then considered to be a group D chromosome) in rare patients with bilateral retinoblastoma and presenting mental retardation and a dysmorphic syndrome suggested that the retinoblastoma susceptibility gene was localized in this chromosomal region (Baud et al., 1999, Lele et al., 1963). Comparative analysis of highly polymorphic germline and tumour genetic markers localized in 13q14 subsequently demonstrated loss of heterozygosity in about $65 \%$ of tumours. In other words, in more than one half of tumours, the susceptibility gene is altered in somatic cells by complete loss of the chromosomal region in which it is localized. It has also been demonstrated that, in familial forms of retinoblastoma, the remaining allele in the tumour was always the allele common to all affected members of the family, i.e. the allele carrying the predisposition to retinoblastoma. Analysis of a large number of retinoblastomas identified the smallest common region of deletion in 13q14, which allowed research to be focussed on this region. In 1986, identification of a gene localized in the region of interest and constituting a site of inactivating germline mutations in children with bilateral retinoblastoma confirmed that this gene corresponded to the retinoblastoma susceptibility gene; it was called RB1(Friend et al., 1986). Identification of $R B 1$ confirmed the complementary hypotheses of Knudson and Comings, opened the way to cancer susceptibility gene testing and allowed definition of the risk of retinoblastoma within particular families.

The RB1 gene codes for a $110 \mathrm{kD}$ nuclear protein with an ubiquitous expression, which, together with proteins p107 and p130, belongs to the pocket protein family. These proteins share in common a domain corresponding to a highly conserved region, the pocket domain, which allows sequestration of transcription factors, such as those of the E2F family. During the G1/S transition of the cell cycle, the pRB protein binds to E2F factors and suppresses their activity, consequently blocking progression to $S$ phase. Inversely, phosphorylation of pRB releases E2F factors, allowing completion of the cell cycle. The pRB protein is involved not only in regulation of the cell cycle, but also in control of termination of cellular differentiation and in exit of the cell from the cell cycle during development. It appears to interact with more than 100 different proteins (Zhu, 2005, Classon\&Harlow, 2002, Chau\&Wang, 2003, Bremner et al., 2004). It is probably this role in differentiation which explains the spatiotemporal specificity of the tumour risk associated with RB1 gene mutations and consequently damage to retinal neuroectodermal cells during early childhood. 


\section{Genetic counseling protocols}

\subsection{Overview}

Whenever unilateral or bilateral retinoblastoma is diagnosed in a child, it is important to consider the possibility of a genetic predisposition and therefore the risk of development of the disease in young children related to the patient.

Analysis of the family history and the tumour history of a patient treated for retinoblastoma is essential to evaluate the possibility of a genetic predisposition and the risk of development of the disease in other members of the child's family and to guide the ophthalmological surveillance of family members (siblings, cousins or offspring). It is therefore possible to calculate the probability of relatives of a child with retinoblastoma to present a genetic predisposition to this disease. These calculations are based on the following elements: (1) 100\% of patients with bilateral retinoblastoma and 10\% of patients with unilateral retinoblastoma are considered to present a genetic predisposition, (2) the mode of transmission is dominant; a carrier parent therefore has once chance in two of transmitting the susceptibility gene to each child, (3) the penetrance is $90 \%$ at birth, which means that an adult who did not develop retinoblastoma in childhood has a tenfold lower probability of being a carrier compared to the probability at birth (Figure 1).

Based on comparative analysis of the various approaches to ophthalmological surveillance in different countries and our multidisciplinary experience at Institut Curie, we can propose guidelines for the surveillance of relatives of patients followed for retinoblastoma (Figure 1) (Abramson et al., 1998, Moll et al., 2000, Musarella\&Gallie, 1987). The modalities of this surveillance depend on the probability of predisposition of the child to be followed, which depends on the child's age and degree of kinship with the affected child, and on the age distribution at diagnosis in predisposed children followed since birth. In a series of 50 predisposed children followed since birth, the diagnosis of retinoblastoma was established before the age of 6 months in $80 \%$ of cases, before the age of 18 months in $92 \%$ of cases and at the age of 4 years in one case. Finally, even for the lowest levels of risk for which ophthalmological surveillance is recommended, ocular fundus examination must be performed at least every 3 months until the age of 24 months in order to ensure effective prevention. These surveillance guidelines are very rigorous: ocular fundus examination at the first month of life, or even the first week, in a specialized unit, with frequent follow-up examinations requiring general anaesthesia from the second or third examination. For example, in the case of a $50 \%$ risk of being a carrier (a child born to a patient with bilateral retinoblastoma), surveillance starts at the first week of life, and then once a month until the age of 18 months (Figure 1).

These guidelines must be maintained in the absence of genetic testing or while waiting for the results, as genetic testing in all patients with unilateral or bilateral retinoblastoma, followed by testing of the relatives, can eliminate the need for surveillance of a certain number of children, depending on the results.

\subsection{Counseling}

Molecular genetic studies of the RB1 gene can now be proposed to all patients with familial or sporadic unilateral or bilateral retinoblastoma. Genetic testing must be performed in the 


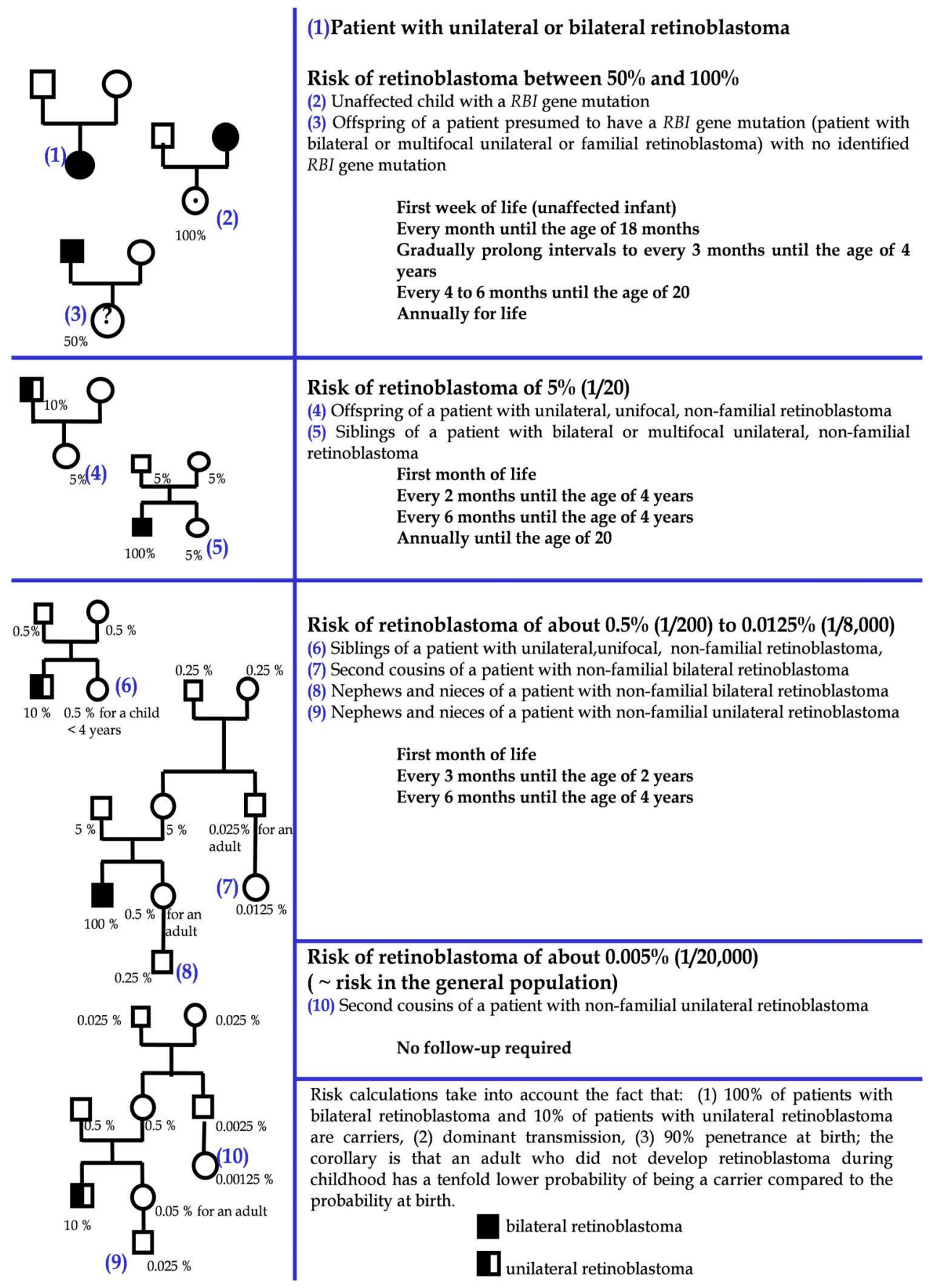

Fig. 1. Ophthalmological surveillance guidelines 
context of a genetics consultation in collaboration with the ophthalmology, paediatric oncology and radiotherapy teams managing the child. During this consultation, the patient's pedigree is built looking for other tumour cases in the family and especially other retinoblastoma cases. Patient or parents of young patients are informed about retinoblastoma predisposition. Ocular fundus examination of parents is required to search for retinoma which would reveal a previously unknown family history. Follow up of young patient's relatives by ocular fundus is recommended. Blood sampling for RB1 molecular analysis is proposed to search for germline mutation. Finally, an informed consent has to be signed by the patients or their legal guardians if RB1 screening is accepted. Following RB1 screening, results are delivered during another genetic consultation. The printed test results are given to the parents and are also kept by the genetics department for at least thirty years, so that they can be consulted by the child during early adulthood. Today, a first-line screening for the two inactivating somatic mutations in the tumor DNA (when available) is performed and represents an attractive alternative: identification of these mutations only in the tumour and not in the leukocytes of the patient eliminates the risk of recurrence in siblings and cousins (see below).

The assessment usually starts with molecular genetic testing but cytogenetic analysis is performed as first-line procedure in the case of associated mental retardation or characteristic dysmorphic syndrome.

\subsection{Clinical management/surveillance (Figure 2)}

When a mutation has been demonstrated in an affected child, genetic testing based on screening for this mutation, is recommended for the siblings. Ophthalmological surveillance can be stopped in a relative when genetic testing fails to reveal the mutation identified in the family. Genetic testing is also proposed to the parents. If one of the two parents carries the mutation, antenatal diagnosis may be proposed for a subsequent pregnancy. If the parents do not carry the mutation, their respective families can be reassured, eliminating the need for ophthalmological surveillance of the patient's cousins. In contrast, it is impossible to assess the level of representation of the mutation identified in the affected child in the gametes of the parent in which a de novo mutation has occurred (quantification of the germline mosaic), or, in other words, it is impossible to eliminate the risk of recurrence in the siblings of the affected child. In this case, for each new birth in the immediate family, a genetic test must be proposed during the neonatal period. Antenatal diagnosis can be proposed case by case.

When no RB1 gene mutation is demonstrated in the affected child:

1. In the case of bilateral retinoblastoma, genetic screening techniques have certain limitations and may fail to demonstrate a mutation, in which case surveillance of the patient's relatives must be continued (Figure 1). Somatic mosaics may also be observed, as an alteration of the RB1 gene can occur in the patient during embryonic development and may not be present in leukocyte DNA. If the mutation is present in the germline, this patient may transmit the mutation to his/her offspring. It is currently proposed to repeat $R B 1$ gene testing at the birth of each child of a patient with a history of bilateral retinoblastoma in childhood in whom molecular RB1 gene testing was negative. 


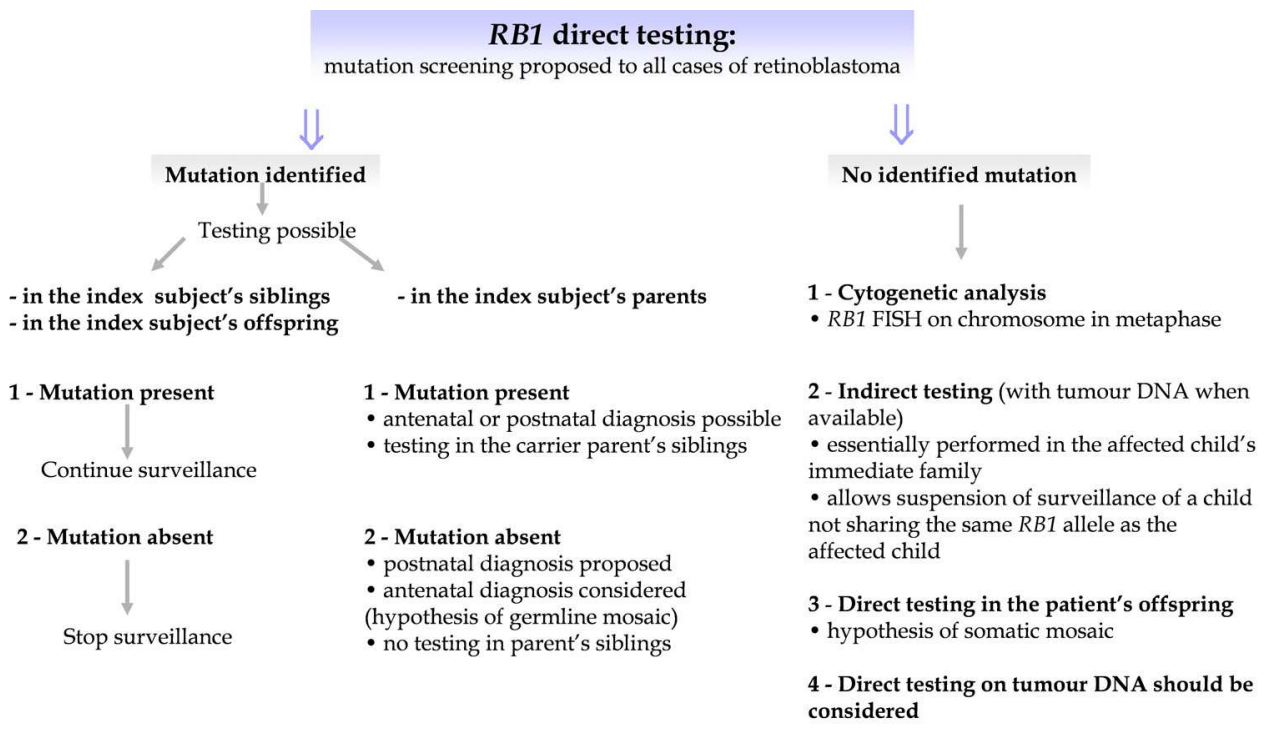

\section{$R B 1$ indirect testing: \\ Analysis of transmission of intragene and extragene markers of the RB1 gene}

1 - Proposed immediately in the case of familial retinoblastoma with two accessible cases

2 - Proposed when no mutation is identified on direct testing or while waiting for the result of direct testing

Fig. 2. Clinical management/surveillance

2. In the case of unilateral sporadic retinoblastoma, the genetic counsellor can be more reassuring, as the risk of a genetic predisposition is very low ( $1 \%$ instead of $10 \%$, taking into account a $90 \%$ screening sensitivity, see below). However, once again, certain limitations of the techniques used and the risk of somatic mosaic must be kept in mind. It is therefore recommended to continue ophthalmological surveillance in the patient's offspring (Figure 1). However, if the probability of predisposition of a child with unilateral retinoblastoma is only $1 \%$, the risk for his nephews is around $0,00125 \%$ or $1 / 80000$ i.e. lower than in the general population. As a result their ophtalmologic follow-up should be stopped.

3. In familial forms comprising two accessible cases, indirect genetic testing rapidly demonstrates the mutant allele of the RB1 gene. This method can then be used to detect relatives with the cancer-predisposing allele and allows the possibility of antenatal diagnosis. Indirect molecular genetic testing can also be proposed for families with only one case of retinoblastoma, while waiting for the results of RB1 screening or when no mutation is detected. The objective in this setting is to suspend surveillance of a child not sharing any RB1 allele in common with its brother or sister with retinoblastoma, i.e. in one case in four, or even one case in two when loss of heterozygosity is demonstrated in the patient's tumour, designating the remaining allele as the putative predisposing allele. It should be stressed that even when a child shares an allele in common with the patient, the probability that he or she has an RB1 gene mutation remains very low (Figure 1). However, as a precaution, 
ophthalmological surveillance should be continued. Antenatal diagnosis cannot be considered in this particular setting.

\subsection{Notes}

For a long time, it was considered that all patients with a deleterious $R B 1$ gene mutation developed retinoblastoma regardless of the type of molecular lesion. However, the risk within a given family has now been clearly established to be heterogeneous, as some members do not develop retinoblastoma, while others develop bilateral retinoblastoma, or even a secondary tumour. The severity of the risk can be evaluated by the disease-eye-ratio (DER), which is a good marker of penetrance and level of expression (Lohmann et al., 1994). The DER is the ratio of the number of eyes affected over the number of carriers within the family. One of the problems of genetic counseling for retinoblastoma is therefore to evaluate the tumour risk for an unborn child with a germline $R B 1$ gene mutation, hence the importance of developing our knowledge of genotype-phenotype relationships.

In general, subjects with a mutation in the first generation may have an attenuated phenotype due to a possible mosaic. The type of lesion then varies according to the type of mutation (Lohmann\&Gallie, 2004, Harbour, 2001, Taylor et al., 2007).

Subjects with a mutation leading to a truncated protein (stop, frameshift) have a high risk, greater than $90 \%$, of bilateral retinoblastoma (mean DER $=1.85$ ). Of note, some truncating mutations in exon 1 may lead to low-penetrance retinoblastoma trough alternative translation initiation (Sanchez-Sanchez et al., 2007). The situation is more complex for the other types of mutations, as discussed below.

Splicing mutations are associated with a lower mean DER (1.5) and, in some cases, with high intrafamily variability with the presence of tumour-free and bilateral cases in the same family. The variability of the DER is mainly due to maintenance of the frame and/or the respect of functional domains. The case of IVS06+1G>T splicing mutation is quite remarkable, as this mutation is supposed to result in a skip of exon 6 out of phase and therefore in the absence of protein, as the truncated messenger is eliminated by Non sense Mediated Decay (NMD) (Holbrook et al., 2004). This mutation is actually associated with an extraordinary variability of intrafamily and interfamily penetrance. The mechanisms proposed to account for this phenomenon are maintenance and therefore translation of the truncated messenger, possibly related to a parental effect (Klutz et al., 2002) or overexpression of the wild-type allele, resulting in a normal level of $R B 1$ expression (Taylor et al., 2007).

Anomalies of the promoter region are classically associated with variations of the level of expression of the messenger and result in variable but generally low DER.

Missense mutations are very rare. When they do not alter splicing (see above), they can be responsible for a partially functional protein (e.g. R661W), which results in a very low mean DER (0.3), but the possibility of bilateral cases cannot be excluded (Onadim et al., 1992).

Chromosomal rearrangements (deletion or duplication of one or several exons, or even the whole gene) are associated with a variable DER (mean: 1.4), particularly and surprisingly, deletions comprising all of $R B 1$, for which the phenotype can vary from no lesion to bilateral retinoblastoma (Albrecht et al., 2005). 
The type of mutation therefore affects the type of lesion, but modifying factors influencing splicing, the level of expression and/or cell survival also appear to be involved. As an example, the existence of these genetic modifiers in retinoblastoma have been suspected and searched in the pRB or p53 pathways in which MDM2 is a key regulator of both p53 and pRB catabolism. We have recently demonstrated that the minor allele of MDM2 that includes a 309T $>\mathrm{G}$ transversion (SNP rs2279744) in the MDM2 promoter is strongly associated under a recessive model with incidence of bilateral or unilateral retinoblastoma among members of retinoblastoma families (Castéra et al., 2010).

In the context of genetic counseling, the possibility of antenatal or even pre-implantation diagnosis can be proposed to couples with a 50\% risk of transmitting an RB1 gene mutation. The situation is obviously more delicate in families presenting an intrafamily heterogeneous risk, which makes genetic counseling more difficult. Although it appears impossible to reassure a parent with no history of retinoblastoma, but carrying an RB1 mutation about the tumour risk for his/her offspring, it is very difficult to inform this subject about techniques allowing the birth of a mutation-free infant. It is therefore very important to continue the study of these families in order to improve genetic counseling in the context of retinoblastoma.

\section{Molecular methods in genetic testing}

\subsection{Overview}

The molecular pathology of $R B 1$ is very diverse and about 500 distinct germline mutations have been described to date, some of which are listed in two databases managed by Dr Lohmann (http://RB1-lsdb.d-lohmann.de) and Dr Pestaña (http://www.es.embnet.org/ Services/MolBio/rbgmdb). These mutations occur throughout the coding sequence and in the promoter region with the notable exception of the last 2 exons (figure 3). Most of these

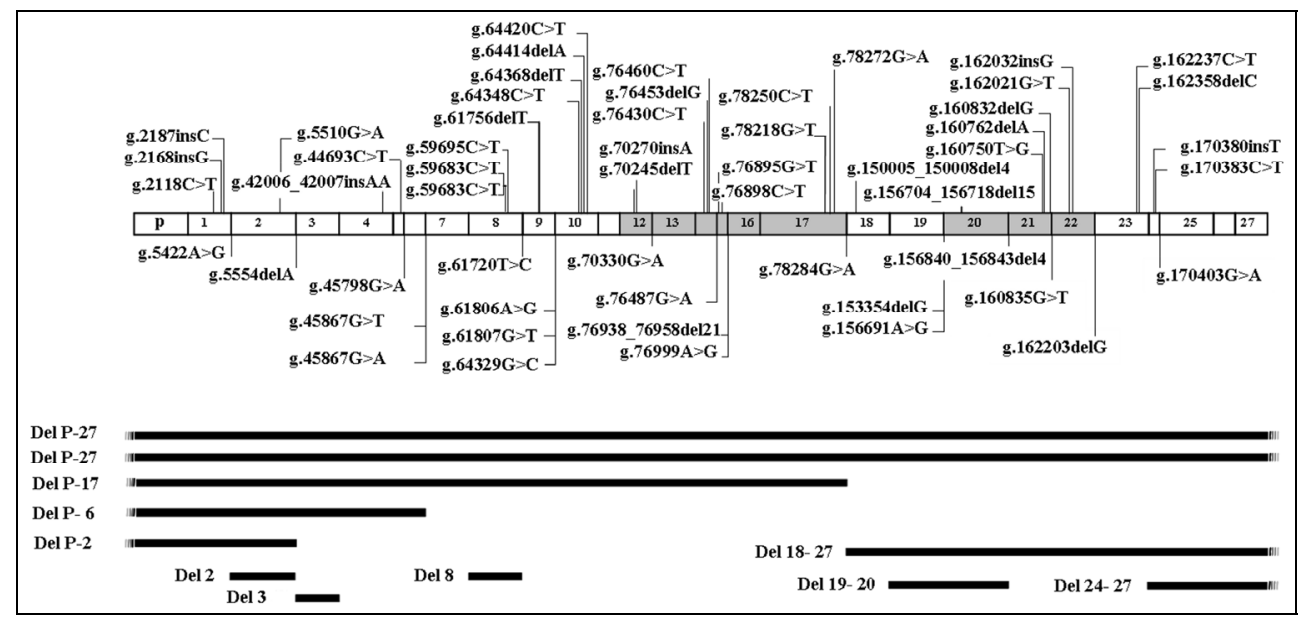

RB1 coding sequence is drawn to scale, and exons shown in grey are part of pocket domains A or B. Large deletions are represented as black lines.

Fig. 3. Pattern of mutations found in a series of 192 retinoblastoma patients (adapted from Houdayer et al., 2004) 
mutations are de novo mutations. The spectrum of germline mutations mainly comprises nonsense mutations (about 40\%), frameshift insertions or deletions of several bases (about $25 \%$ ), altered splicing (about 20\%) and chromosomal rearrangements, i.e. deletions/duplications of one or several exons, or even the entire gene (about $10 \%$ ). The remaining mutations correspond to rare missense mutations and mutations of the promoter region. Variations of the relative proportions of these mutations have been reported, which can be explained by differences of the population studied, and environmental or stochastic factors, related to the high rate of de novo mutations (Dehainault et al., 2004, Alonso et al., 2001). Finally, constitutional inactivation of RB1 can be due to exceptional cases of chromosomal rearrangements only visible on cytogenetics (e.g. translocations, inversions). These same types of alterations are also found in the tumour, as well as hypermethylation of the promoter region and large chromosomal losses comprising all of RB1 and flanking regions (Richter et al., 2003).Tumoral events are now systematically searched when the tumor is available.

\subsection{Materials}

Analysis of the index case, which requires a larger amount of material, must be distinguished from that of relatives, in whom the search for a previously identified mutation requires less material.

\subsubsection{Study of the index case}

Testing for germline RB1 gene mutations is classically performed on DNA extracted from whole blood collected on EDTA. Two to $3 \mu \mathrm{g}$ of genomic DNA are required for screening for point mutations and large mutations on the entire gene. Extraction can be performed with commercial kits or by phenol/chloroform or perchlorate/chloroform or salting out techniques (Johns\&Paulus-Thomas, 1989, Miller et al., 1988).

When DNA is used for screening, RNA must also be available due to the frequency of splicing alterations. RNA analysis may be essential to demonstrate the impact of the presumptive mutation identified on genomic DNA. RNA is obtained from a blood sample collected on heparin, Acid Citrate Dextrose (ACD), or EDTA. Lymphoblastoid cell culture is an interesting option, as it provides an infinite source of nucleic acids, but it is expensive and requires cell culture facilities.

Finally, tumour DNA analysis is important for the molecular diagnosis of retinoblastoma. Samples fixed in Bouin's solution cannot be used, as this fixative degrades DNA, and frozen blocks are preferable to paraffin-embedded blocks. When the first-line analysis is performed on the tumour (see below), a sufficient amount of material must be available ( 2 to $3 \mu \mathrm{g}$ ). A small quantity is sufficient when looking for a known mutation and it is even possible to obtain genetic material by scratching a slide. Tumour DNA can be extracted with commercial kits or phenol/chloroform.

\subsubsection{Study of relatives}

In this setting, genetic testing is designed to detect a previously identified mutation, and a small quantity of DNA is sufficient (about $50 \mathrm{ng}$ ) and can be extracted from buccal cells 
collected by swabbing, a simple, noninvasive technique. FTA technology (Gaytmenn et al., 2002, Seah et al., 2003) is then the preferred method: swabs are applied onto FTA cards, buccal cells are then lysed and nucleic acids are immobilized and stabilized in the FTA matrix. The paper support is then punched out and the punch is washed and placed in the PCR reaction tube. Alternatively, buccal cells can be extracted from the swab by using standard commercial kits.

\subsection{Methods}

There are two types of diagnostic genetic molecular testing: direct testing and indirect testing.

\subsubsection{Direct testing}

Direct testing consists of looking for a germline alteration of the $R B 1$ gene indicative of a predisposition to retinoblastoma. The first study performed in a family is time-consuming and generally takes several months. In contrast, when testing is performed to detect a mutation already identified in the family, targeted screening of the previously identified mutation takes only a few days. Direct testing is essentially performed on blood samples. It is good practice to verify the presence of a mutation on two DNA samples obtained independently: two blood samples taken at two different times, or a blood test and a buccal swab.

Direct testing can also be performed on the tumour. This screening can be very useful in bilateral cases in which no mutation is detected on leukocytes, but also for unilateral forms, as identification of a mutation only in the tumour would be very useful for genetic counselling, eliminating the risk of recurrence in siblings and cousins (but not in the offspring). Testing of tumour DNA is obviously subject to availability of material, i.e. enucleation.

The complexity of the mutational spectrum of the $R B 1$ gene requires analysis of the entire coding sequence and promoter region by several complementary techniques (see DNA methylation analysis).

\subsubsection{Detection of point mutations}

Point mutations are usually investigated by Denaturing High Performance Liquid Chromatography (Xiao\&Oefner, 2001, Dehainault et al., 2004) and/or direct DNA sequencing (Richter et al., 2003), or even Denaturing Gradient Gel Electrophoresis (which is more complicated to perform) (Fodde\&Losekoot, 1994) or Single Strand Conformational Polymorphism (low sensitivity) (Orita et al., 1989). Recently, we have adapted a novel HDA method (Houdayer et al., 2010) called Enhanced Mismatch Mutation Analysis (EMMA).

Regardless of the technique used, the gene is cut into amplicons corresponding to the exon sequence and intron/exon junctions in order to detect any abnormalities of splicing consensus sequences which can have major functional consequences.

DHPLC is an adaptation of high performance liquid chromatography for DNA applications, used for the detection of point mutations. It is based on the principle of physical separation, under denaturing conditions, of various DNA fragments in a mobile phase by differential 
retention on a solid phase composed of a DNA column. DHPLC has a high sensitivity, making it a very useful tool in retinoblastoma for the study of tumours or mosaics, which are not uncommon. DHPLC is able to detect less than $20 \%$ of the minority allelic species, which corresponds to the accepted limit for sequencing. The limitations of DHPLC depend on the base composition of the DNA fragment studied (see "Notes" section).

EMMA is based on the use of innovative matrices increasing the electrophoretic mobility differences between homoduplex and heteroduplex. DNA Sensitivity is further improved by using nucleosides as additives to enhance single-base substitution detection. Nucleosides are expected to interact with mismatched bases of heteroduplexes, thereby increasing mobility differences with homoduplexes. Moreover, this method, in combination with adapted semiquantitative PCR conditions, can be used to simultaneously detect point mutations and large-scale rearrangement in a single run (Weber et al., 2006, Weber et al., 2007). This feature, combined with the use of a single set of separation conditions for all fragments and with the multiplexing capability of the method, leads to a considerable simplification and cost reduction compared to previous methods (Caux-Moncoutier et al., 2010).

Direct sequencing is the second option and is considered to be the reference technique. However, its performances are highly dependent on the apparatus, chemistry, polymers and software used. A study comparing DHPLC and direct sequencing for BRCA1 analysis concluded on a similar detection rate for the two techniques (Alonso et al., 2001).

Direct testing is also performed on RNA to characterize any abnormal splicing. Classically, after extraction of RNA and RT PCR, the cDNA region surrounding the putative anomaly is amplified to demonstrate abnormal transcripts. The instability of messenger RNA carrying a premature stop codon, or NMD (Holbrook et al., 2004), constitutes a real problem in diagnostic molecular genetics and is discussed in the "Notes" section.

\subsubsection{Detection of chromosomal rearrangements}

Chromosomal rearrangements, i.e. deletion/duplication of one or several exons, cannot be detected by the techniques used to detect point mutations because the mutant allele is masked by the wild-type allele, as the retinoblastoma susceptibility gene segregates according to an autosomal dominant mode.

Specific gene assay techniques must be used in order to distinguish 2 copies of the target (wild-type status), one copy (deletion) or 3 copies (duplication). Semiquantitative techniques, such as Quantitative Multiplex PCR of Short fluorescent Fragments (QMPSF), Multiplex PCR/liquid chromatography assay (MP/LC) (Duponchel et al., 2001, Dehainault et al., 2004), and Multiplex Ligation Probe Amplification (MLPA) (Schouten et al., 2002) or quantitative techniques, i.e. real-time PCR, are used. QMPSF is a technique allowing simultaneous, semiquantitative amplification of several exons; the intensity of the signal obtained therefore depends on the number of copies of the gene of interest in the matrix DNA. After amplification of the exons followed by separation of the PCR products obtained by electrophoresis, the patient electrophoretograms are compared to those of normal and mutant controls. The signal intensity in the various samples is then evaluated and deletions of one or several amplicons are revealed by a $50 \%$ reduction of the corresponding peak(s). Data can be exported to an Excel spreadsheet and analysed by a macro. The advantage of 
QMPSF and other semiquantitative multiplex PCR approaches is their high throughput and the small number of analytical steps, which is always appreciated in the diagnostic setting.

Another widely used semiquantitative technique, MLPA, is based on a step of hybridization of specific probes, fitted with a universal tail, and corresponding to the exons to be examined. The quantity of probe hybridized is therefore proportional to the quantity of target. After hybridization and ligation, probes are then amplified by PCR with a set of universal primers and PCR products are separated be capillary electrophoresis. Once again, the signal intensity, compared to that of normal and mutant controls, is used to detect chromosomal rearrangements. Despite a much higher multiplexing capacity, the throughput of MLPA is lower than that of semiquantitative multiplex PCR approaches due to an additional analytical step (ligation), but the advantage of this technique is that it is available in the form of ready-for-use kits for many genes including $R B 1$.

These two approaches have similar performances and the choice between the two therefore depends on the user's priorities.

Real-time PCR techniques are particularly suitable for gene assays. They are based on either i) incorporation of a free fluorophore (typically SYBR Green) into the forming strands, which generates an increase in the intensity of fluorescence with the number of copies produced. The sensitivity of these techniques is enhanced by the use of fluorophore-labelled specific probes. Unfortunately, they have a low throughput, limited by the number of fluorophores available and are therefore not widely used for screening, especially as this low throughput is not justified by the gain in sensitivity.

An approach combining the search for point mutations and chromosomal rearrangements achieves a germline RB1 gene mutation detection rate of about $90 \%$ among patients with bilateral retinoblastoma (Richter et al., 2003). The mutations that are not detected are probably deep intronic anomalies, responsible for alternative splicing defects that are not detected because they are situated outside of the zones usually studied (Dehainault et al., 2007). They can also correspond to mosaics, which cannot be detected on circulating leukocytes (see "Notes" section).

\subsubsection{DNA methylation analysis}

Hypermethylation of the promoter region is a common mutational event found in tumor (Richter et al., 2003). Hypermethylation of the promoter are investigated by bisulfite analysis followed either by sequencing, by methylation-specific PCR or by a quantitative analysis of methylated allele using specific Taqman ${ }^{\circledR}$ probes (De La Rosa-Velazquez et al., 2007, Richter et al., 2003, Zeschnigk et al., 2004). Alternatively, tumor DNA can be digested using methylation sensitive enzyme (CfoI as an example), followed by PCR amplification of the promoter and agarose gel electrophoresis or followed by a semiquantitative technique such as QMPSF (Taylor et al., 2007).

\subsubsection{Indirect testing}

Indirect testing is based on amplification of polymorphic markers of the RB1 locus. Analysis of polymorphic genetic markers localized in and around the $R B 1$ gene in an affected child and his parents is designed to identify the RB1 allele carrying or putatively carrying a predisposition to retinoblastoma. Indirect tests are very useful in familial cases, when 
samples are available for at least two cases of retinoblastoma, to identify the allele of the RB1 gene common to affected cases, i.e. to determine the allele that carries the mutation, even when the mutation has not been demonstrated directly. In non-familial forms, reconstitution of the affected child's alleles reveals the two alleles putatively associated with an alteration of the RB1 gene. Tumour DNA studies (when available) reveal loss of an allele in $65 \%$ of cases and consequently allow identification of the remaining allele, i.e. potentially carrying a germline mutation. The indirect approach is technically simple and rapid, but nevertheless requires testing of the affected child, the parents, and possibly other relatives.

\subsubsection{Cytogenetic analysis}

Cytogenetic analysis comprises a standard karyotype and analysis of the $R B 1$ gene by FISH or CGH-array. The development of molecular genetic studies has considerably limited the applications of cytogenetics and its only first-line indication is for karyotyping in a child with mental retardation or a malformative syndrome associated with retinoblastoma. However, it remains useful for the detection of translocations and mosaic deletions and can help to estimate the size of very large deletions. It therefore reveals certain rare situations.

High density CGH array may be useful for fine mapping of deletion breakpoints in a context of a contiguous gene syndrome (Mitter et al., 2011)

\subsection{Notes}

\subsubsection{General problems}

\subsubsection{GC-rich regions}

The $5^{\prime}$ part of the RB1 gene (promoter and exon 1) is particularly rich in GC, which can make it difficult to analyse, for the detection of both point mutations and rearrangements. Due to the high degree of similarity of the amplified region, nonspecific intrastrand base pairing tends to occur during PCR, resulting in nonspecific PCR. Consequently, some teams do not analyse this region, which makes the analysis incomplete, as mutations of the promoter region and exon 1 have been well documented. We have resolved these problems by the addition of dimethylsulfoxyde (DMSO) to the reaction medium.

\subsubsection{Mosaics}

The existence of somatic mosaics in retinoblastoma raises a major problem for molecular diagnosis, as the mutant clone may be below the limit of detection of the technique used, or may even be absent from the cells studied. We have identified a deleterious RB1 mutation from a blood sample of an affected child in whom tumour material was not available. Surprisingly, this mutation was detected in a very small percentage (estimated at $10 \%$ ) of buccal cells. DHPLC is particularly useful in this context because of its high sensitivity, but characterization of the anomaly by sequencing remains problematical and requires specific techniques (fraction collector, specific primers, cloning, etc.).

\subsubsection{Abnormalities of splicing}

Splicing abnormalities represent $20 \%$ of the mutational spectrum of $R B 1$ and are therefore important to characterize. Unfortunately, the instability of messenger RNA carrying a premature stop codon means that the truncated messenger RNA is below the limit of 
detection and only the wild-type transcript will be visible, wrongly suggesting the absence of an anomaly. This problem can sometimes be resolved by using translation inhibitors, such as puromycin, which eliminate NMD and therefore improve detection of the truncated messenger RNA (Andreutti-Zaugg et al., 1997).

Some deep intronic anomalies, responsible for alternative splicing, are probably not detected because they are situated outside the zones routinely studied. Systematic RNA analysis would be required to demonstrate these anomalies. This approach, unsuitable for routine testing, is nevertheless used in the case of failure of other techniques in situations of highly probable predisposition, such as cases of bilateral and/or familial retinoblastoma with no identified mutation (Dehainault et al., 2007).

\subsubsection{Detection of point mutations by DHPLC}

DHPLC is a sensitive and reliable technique for the detection of point mutations. However, its efficacy is subject to the availability of specific, high-yield PCR and rigorous system quality control, as the gradient drift can impair the quality of testing by modifying retention times and, much more insidiously, loss of calibration, even minimal, of the oven can be responsible for a drastic reduction of resolution for certain amplicons, generating falsenegative results. It is therefore essential to ensure the integrity of the system each day by using control samples. Control samples should generate slight modifications of the profile, which can therefore only be detected with an optimally functioning system. The limitations of this method, which depend on the base composition of the DNA fragment studied, must be kept in mind. For example, despite all of our efforts, we have not been able to obtain reliable results for exon 8 of $R B 1$, which must be sequenced as first-line procedure (Dehainault et al., 2004). This point has also been emphasized by P. Oefner, the inventor of DHPLC (Sivakumaran et al., 2005).

\subsubsection{Detection of chromosomal rearrangements by semiquantitative techniques}

MLPA or semiquantitative multiplex PCR techniques (such as QMPSF) are robust techniques, but highly dependent on the quality of the DNA studied. Degraded DNA will be responsible for loss of proportionality between signal intensity and copy number, particularly for large fragments, making the analysis uninterpretable. Contamination of DNA by phenol will have an even greater effect, because it generates a random fluctuation of signal intensity. Phenol-free extraction techniques should therefore be preferred (perchlorate/chloroform or column-based commercial kits) or a system ensuring the absence of contamination by phenol such as the gel lock extraction system, which uses a gelbarrier system (Eppendorf®).

It is also essential to adjust all DNA samples studied to a suitable working concentration, classically $50 \mathrm{ng} / \mu \mathrm{l}$. If the DNA concentration is too high, for example, the proportionality between signal intensity and copy number will be lost, particularly for small fragments. DNA calibration can be performed with: i) a tube spectrophotometer (unsuitable for large series); ii) the NanoDrop from NanoDrop technologies (which has the advantage of tracing the spectrum of the sample); or iii) a plate reader (rapid, but reading at only one wavelength at a time). In our experience, the use of fluorescent dyes for the assay, such as PicoGreen (Molecular Probes), is unnecessary for these applications. 
Finally, buccal swabs are poorly adapted to these analyses, including for the search of a mutation already identified in a relative, as DNA is often present in a low concentration and difficult to calibrate for FTA samples.

Due to the importance of quality/quantity/calibration of DNA solutions, laboratories often prefer to extract DNA locally and therefore ask to receive whole blood.

A classical trap in the interpretation of these techniques concerns the false-positive results generated by a PCR primer mismatch. Each deletion of a single exon must therefore be systematically checked by another technique and/or by shifting the primers (long range PCR, RNA studies, real-time PCR, for example). Finally, duplication of an isolated exon is the most difficult case to characterize. The ideal situation is therefore to have a duplicated control of the entire RB1, for example DNA from a case of trisomy 13.

\section{Conclusion}

Finally, we recommend a systematic $R B 1$ molecular screening to all retinoblastoma patients as part of routine clinical care. Emphasis is placed on close collaboration between laboratory staff and clinicians to ensure effective communication and therefore adequate genetic counseling.

\section{Acknowledgments}

We thank Christine Levy, Livia Lumbroso, Jean Michon, Isabelle Aerts, Gudrun Schleiermacher, Daniel Orbach, Hélène Pacquement, Jérôme Couturier, Catherine Dehainault, Dorothée Michaux for participating in Institut Curie Retinoblastoma pluridisciplinary group. This work was supported by grants from the Programme Incitatif et Coopératif sur le Rétinoblastome (Institut Curie) and RETINOSTOP.

\section{References}

Abramson DH, Mendelsohn ME, Servodidio CA, et al. (1998). Familial retinoblastoma: where and when? Acta Ophthalmol Scand 76, pp 334-8

Albrecht P, Ansperger-Rescher B, Schuler A, et al. (2005). Spectrum of gross deletions and insertions in the RB1 gene in patients with retinoblastoma and association with phenotypic expression. Hum Mutat 26, pp 437-45

Alonso J, Garcia-Miguel P, Abelairas J, et al. (2001). Spectrum of germline RB1 gene mutations in Spanish retinoblastoma patients: Phenotypic and molecular epidemiological implications. Hum Mutat 17, pp 412-22

Andreutti-Zaugg C, Scott RJIggo R. (1997). Inhibition of nonsense-mediated messenger RNA decay in clinical samples facilitates detection of human MSH2 mutations with an in vivo fusion protein assay and conventional techniques. Cancer Res 57, pp 3288-93

Baud O, Cormier-Daire V, Lyonnet S, et al. (1999). Dysmorphic phenotype and neurological impairment in 22 retinoblastoma patients with constitutional cytogenetic $13 q$ deletion. Clin Genet 55, pp 478-82

Bremner R, Chen D, Pacal M, et al. (2004). The RB protein family in retinal development and retinoblastoma: new insights from new mouse models. Dev Neurosci 26, pp 417-34,

Castera L, Sabbagh A, Dehainault C, et al. (2010). MDM2 as a modifier gene in retinoblastoma. J Natl Cancer Inst 102, pp 1805-8 
Caux-Moncoutier V, Castera L, Tirapo C, et al. (2010). EMMA, a cost- and time-effective diagnostic method for simultaneous detection of point mutations and large-scale genomic rearrangements: application to BRCA1 and BRCA2 in 1,525 patients. Hum Mutat,

Chau BNWang JY. (2003). Coordinated regulation of life and death by RB. Nat Rev Cancer 3, pp 130-8,

Classon MHarlow E. (2002). The retinoblastoma tumour suppressor in development and cancer. Nat Rev Cancer 2, pp 910-7

Comings DE. (1973). A general theory of carcinogenesis. Proc Natl Acad Sci U S A 70, pp 3324-8

De La Rosa-Velazquez IA, Rincon-Arano H, Benitez-Bribiesca L, et al. (2007). Epigenetic regulation of the human retinoblastoma tumor suppressor gene promoter by CTCF. Cancer Res 67, pp 2577-85

Dehainault C, Lauge A, Caux-Moncoutier V, et al. (2004). Multiplex PCR/liquid chromatography assay for detection of gene rearrangements: application to RB1 gene. Nucleic Acids Res 32, pp e139

Dehainault C, Michaux D, Pages-Berhouet S, et al. (2007). A deep intronic mutation in the RB1 gene leads to intronic sequence exonisation. Eur J Hum Genet 15, pp 473-7,

Draper GJ, Sanders BMKingston JE. (1986). Second primary neoplasms in patients with retinoblastoma. Br J Cancer 53, pp 661-71

Duponchel C, Di Rocco C, Cicardi M, et al. (2001). Rapid detection by fluorescent multiplex PCR of exon deletions and duplications in the C1 inhibitor gene of hereditary angioedema patients. Hum Mutat 17, pp 61-70

Fletcher O, Easton D, Anderson K, et al. (2004). Lifetime risks of common cancers among retinoblastoma survivors. J Natl Cancer Inst 96, pp 357-63,

Fodde RLosekoot M. (1994). Mutation detection by denaturing gradient gel electrophoresis (DGGE). Hum Mutat 3, pp 83-94

Friend SH, Bernards R, Rogelj S, et al. (1986). A human DNA segment with properties of the gene that predisposes to retinoblastoma and osteosarcoma. Nature 323, pp 643-6

Gaytmenn R, Hildebrand DP, Sweet D, et al. (2002). Determination of the sensitivity and specificity of sibship calculations using AmpF 1STR Profiler Plus. Int J Legal Med 116, pp 161-4

Harbour JW. (2001). Molecular basis of low-penetrance retinoblastoma. Arch Ophthalmol 119, pp 1699-704

Holbrook JA, Neu-Yilik G, Hentze MW, et al. (2004). Nonsense-mediated decay approaches the clinic. Nat Genet 36, pp 801-8

Houdayer C, Gauthier-Villars M, Lauge A, et al. (2004). Comprehensive screening for constitutional RB1 mutations by DHPLC and QMPSF. Hum Mutat 23, pp 193-202

Houdayer C, Moncoutier V, Champ J, et al. (2010). Enhanced mismatch mutation analysis: simultaneous detection of point mutations and large scale rearrangements by capillary electrophoresis, application to BRCA1 and BRCA2. Methods Mol Biol 653, pp 147-80

Johns MB, Jr.Paulus-Thomas JE. (1989). Purification of human genomic DNA from whole blood using sodium perchlorate in place of phenol. Anal Biochem 180, pp 276-8

Klutz M, Brockmann DLohmann DR. (2002). A parent-of-origin effect in two families with retinoblastoma is associated with a distinct splice mutation in the RB1 gene. Am J Hum Genet 71, pp 174-9

Knudson AG, Jr. (1971). Mutation and cancer: statistical study of retinoblastoma. Proc Natl Acad Sci U S A 68, pp 820-3

Lele KP, Penrose LSStallard HB. (1963). Chromosome Deletion in a Case of Retinoblastoma. Ann Hum Genet 27, pp 171-4 
Lohmann DR, Brandt B, Hopping W, et al. (1994). Distinct RB1 gene mutations with low penetrance in hereditary retinoblastoma. Hum Genet 94, pp 349-54

Lohmann DRGallie BL. (2004). Retinoblastoma: revisiting the model prototype of inherited cancer. Am J Med Genet C Semin Med Genet 129, pp 23-8

Miller SA, Dykes DDPolesky HF. (1988). A simple salting out procedure for extracting DNA from human nucleated cells. Nucleic Acids Res 16, pp 1215

Mitter D, Ullmann R, Muradyan A, et al. (2011). Genotype-phenotype correlations in patients with retinoblastoma and interstitial 13q deletions. Eur J Hum Genet 19, pp 947-958, 1476-5438 (Electronic) 1018-4813 (Linking).

Moll AC, Imhof SM, Bouter LM, et al. (1996). Second primary tumors in patients with hereditary retinoblastoma: a register-based follow-up study, 1945-1994. Int J Cancer 67, pp 515-9

Moll AC, Imhof SM, Meeteren AY, et al. (2000). At what age could screening for familial retinoblastoma be stopped? A register based study 1945-98. Br J Ophthalmol 84, pp $1170-2$

Musarella MAGallie BL. (1987). A simplified scheme for genetic counseling in retinoblastoma. J Pediatr Ophthalmol Strabismus 24, pp 124-5

Onadim Z, Hogg A, Baird PN, et al. (1992). Oncogenic point mutations in exon 20 of the RB1 gene in families showing incomplete penetrance and mild expression of the retinoblastoma phenotype. Proc Natl Acad Sci U S A 89, pp 6177-81

Orita M, Suzuki Y, Sekiya T, et al. (1989). Rapid and sensitive detection of point mutations and DNA polymorphisms using the polymerase chain reaction. Genomics 5, pp 874-9

Richter S, Vandezande K, Chen N, et al. (2003). Sensitive and efficient detection of RB1 gene mutations enhances care for families with retinoblastoma. Am J Hum Genet 72, pp 253-69,

Sanchez-Sanchez F, Ramirez-Castillejo C, Weekes DB, et al. (2007). Attenuation of disease phenotype through alternative translation initiation in low-penetrance retinoblastoma. Hum Mutat 28, pp 159-67,

Schouten JP, McElgunn CJ, Waaijer R, et al. (2002). Relative quantification of 40 nucleic acid sequences by multiplex ligation-dependent probe amplification. Nucleic Acids Res 30, pp e57,

Seah LH, Jeevan NH, Othman MI, et al. (2003). STR Data for the AmpFISTR Identifiler loci in three ethnic groups (Malay, Chinese, Indian) of the Malaysian population. Forensic Sci Int 138, pp 134-7,

Sivakumaran TA, Shen P, Wall DP, et al. (2005). Conservation of the RB1 gene in human and primates. Hum Mutat 25, pp 396-409,

Taylor M, Dehainault C, Desjardins L, et al. (2007). Genotype-phenotype correlations in hereditary familial retinoblastoma. Hum Mutat 28, pp 284-93,

Weber J, Looten R, Houdayer C, et al. (2006). Improving sensitivity of electrophoretic heteroduplex analysis using nucleosides as additives: Application to the breast cancer predisposition gene BRCA2. Electrophoresis 27, pp 1444-52,

Weber J, Miserere S, Champ J, et al. (2007). High-throughput simultaneous detection of point mutations and large-scale rearrangements by CE. Electrophoresis 28 , pp 4282-8,

Xiao WOefner PJ. (2001). Denaturing high-performance liquid chromatography: A review. Hum Mutat 17, pp 439-74,

Zeschnigk M, Bohringer S, Price EA, et al. (2004). A novel real-time PCR assay for quantitative analysis of methylated alleles (QAMA): analysis of the retinoblastoma locus. Nucleic Acids Res 32, pp e125,

Zhu L. (2005). Tumour suppressor retinoblastoma protein Rb: a transcriptional regulator. Eur J Cancer 41, pp 2415-27, 


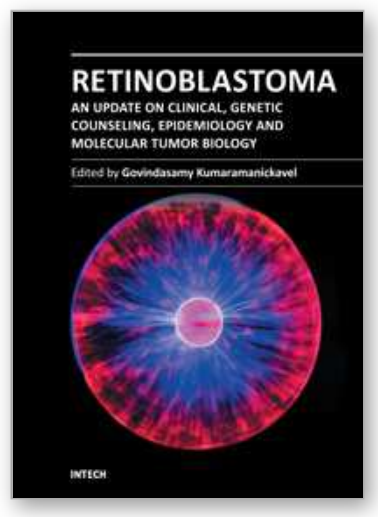

\author{
Retinoblastoma: An Update on Clinical, Genetic Counseling, \\ Epidemiology and Molecular Tumor Biology \\ Edited by Prof. Govindasamy Kumaramanickavel
}

ISBN 978-953-51-0435-3

Hard cover, 170 pages

Publisher InTech

Published online 28, March, 2012

Published in print edition March, 2012

Retinoblastoma is the first tumor suppressor gene discovered ever. The discovery opened a new avenue in the field of oncology leading to the identification of 35 tumor suppressor genes, till date in our genome. This book is an excellent compilation of both clinical and basic science information that meets the needs of a young clinician and a researcher at the same time. It also has abundant information on recent advances and cuttingedge knowledge in intracellular molecular cross-talking of retinoblastoma protein with various cellular viral-like proteins.

\title{
How to reference
}

In order to correctly reference this scholarly work, feel free to copy and paste the following:

Claude Houdayer, Marion Gauthier-Villars, Laurent Castéra, Laurence Desjardins, François Doz and Dominique Stoppa-Lyonnet (2012). Retinoblastoma - Genetic Counseling and Molecular Diagnosis, Retinoblastoma: An Update on Clinical, Genetic Counseling, Epidemiology and Molecular Tumor Biology, Prof. Govindasamy Kumaramanickavel (Ed.), ISBN: 978-953-51-0435-3, InTech, Available from:

http://www.intechopen.com/books/retinoblastoma-an-update-on-clinical-genetic-counseling-epidemiology-andmolecular-tumor-biology/retinoblastoma-genetic-counseling-protocols-and-molecular-diagnostic-protocols-

\section{INTECH}

open science | open minds

\section{InTech Europe}

University Campus STeP Ri

Slavka Krautzeka 83/A

51000 Rijeka, Croatia

Phone: +385 (51) 770447

Fax: +385 (51) 686166

www.intechopen.com

\section{InTech China}

Unit 405, Office Block, Hotel Equatorial Shanghai

No.65, Yan An Road (West), Shanghai, 200040, China

中国上海市延安西路65号上海国际贵都大饭店办公楼 405 单元

Phone: +86-21-62489820

Fax: $+86-21-62489821$ 
(C) 2012 The Author(s). Licensee IntechOpen. This is an open access article distributed under the terms of the Creative Commons Attribution 3.0 License, which permits unrestricted use, distribution, and reproduction in any medium, provided the original work is properly cited. 